\title{
Point-of-Care Testing for Clostridium Difficile Infection: A Real-World Feasibility Study of a Rapid Molecular Test in Two Hospital Settings
}

\author{
Simon D. Goldenberg • Karen N. Bisnauthsing • Amita Patel • \\ Anne Postulka $\cdot$ Duncan Wyncoll $\cdot$ Rebekah Schiff • Gary L. French
}

To view enhanced content go to www.infectiousdiseases-open.com

Received: August 8, 2014 / Published online: September 10, 2014

(C) The Author(s) 2014. This article is published with open access at Springerlink.com

\begin{abstract}
Introduction: In the developed world, Clostridium difficile infection (CDI) is the most important cause of nosocomial infectious diarrhea. In addition to providing epidemiological data and helping to indicate that a local outbreak may be occurring, laboratory tests are used to augment clinical decisions on individual patients. Very rarely do diagnostic tests provide results at the point of

Electronic supplementary material The online version of this article (doi:10.1007/s40121-014-0038-6) contains supplementary material, which is available to authorized users.
\end{abstract}

S. D. Goldenberg $(\bowtie) \cdot$ K. N. Bisnauthsing .

A. Patel · G. L. French

Centre for Clinical Infection and Diagnostics

Research, King's College London and Guy's and St

Thomas' NHS Foundation Trust, Westminster

Bridge Road, SE1 7EH London, UK

e-mail: Simon.goldenberg@gstt.nhs.uk

A. Postulka

Cepheid Europe, Maurens-Scopont, France

D. Wyncoll

Department of Critical Care, Guys and St Thomas'

NHS Foundation Trust, London, UK

R. Schiff

Department of Ageing and Health, Guy's and St

Thomas' NHS Foundation Trust, London, UK decision making; in the intervening period between requesting investigations on a patient with suspected CDI and return of the laboratory result, decisions must be made regarding patient isolation and treatment.

Methods: A 22-month, real-world feasibility study was conducted in patients with clinically significant diarrhea, in a London Hospital between March 2011 and January 2013, in three older persons' wards and two intensive care units (ICUs) to determine acceptability, ease of use, change in turnaround time and clinical utility of a rapid, polymerase chain reaction (PCR)-based point-of-care test (POCT) (Cepheid GeneXpert $^{\circledR}$, Sunnyvale, California, USA) for diagnosis of Clostridium difficile. Nurses in the older persons' ward and laboratory technicians in the ICU were trained to perform the test. Residual samples were sent to the centralized laboratory for parallel testing using a two-step algorithm.

Results: A total of 335 samples were tested using the POCT with a median turnaround time of $1.85 \mathrm{~h}$ compared with $18 \mathrm{~h}$ for the centralized laboratory test. Overall agreement with centralized laboratory testing was $98.1 \%$. Discrepant samples were more frequent on 
elderly wards than ICU. Overall 20/335 (6\%) processing errors were encountered and were highest in the first few months of the study. Significantly more processing errors occurred on the older persons' wards $13 / 102(12.7 \%)$ than on ICU $7 / 271$ (2.6\%). Older persons' patients who had POCT were significantly less likely to have a test requested for bacterial stool culture (3.1\% vs. $10.9 \% p=0.044)$. This difference was not observed in the ICU patients. No other differences in ancillary test requesting, mortality or length of stay were observed.

Conclusions: The majority of users reported that the POCT was easy to perform and was an acceptable part of their job. POCT using this system is feasible and acceptable to nursing staff and technicians working within these two hospital-based settings.

Keywords: Clostridium difficile; Infection; Nosocomial infectious diarrhea; Point-of-care testing; Rapid diagnostics

\section{INTRODUCTION}

Diarrhea is a common symptom in hospitalized patients; however, the majority of patients have a non-infectious etiology [1]. In the developed world, Clostridium difficile infection (CDI) is the most important cause of nosocomial infectious diarrhea [2]. In addition to providing epidemiological data and helping to indicate that a local outbreak may be occurring, laboratory tests are used to augment clinical decisions on individual patients. Very rarely do diagnostic tests provide results at the point of decision making; in the intervening period between requesting investigations on a patient with suspected CDI and return of the laboratory result, decisions must be made regarding patient isolation and treatment.
The average time taken to test for CDI in one study was 1.8 days [3], although other centers performing testing three times per day report turnaround times of $8 \mathrm{~h}$ [4]. The authors have previously reported a median turnaround time of $17.3 \mathrm{~h}$ in their institution's laboratory [1]. As a consequence of diagnostic delays, patients are often presumptively isolated and treated for CDI empirically. For those patients who ultimately test positive, this may be beneficial in terms of preventing cross transmission [5] and improving clinical outcomes; however, isolating a patient with diarrhea due to a non-infectious cause may be wasteful of scarce resources. Similarly, empirical anti-C. difficile treatment may be detrimental to patients. Other studies have found that as much as $40-62 \%$ of empirical therapy for $C$. difficile is inappropriate $[3,6]$. Thus, there is a clinical need for a rapid diagnostic test that can help clinicians make informed decisions quicker, minimizing waste and potentially improving clinical outcomes.

The majority of microbiology tests are performed in centralized laboratories; however, delays are encountered due to two main bottlenecks: transportation to the laboratory and batching of specimens to be tested. There has been a recent trend towards centralization and consolidation of pathology services, which can adversely affect turnaround times $[7,8]$. These problems may be partially resolved by the use of point-ofcare tests (POCT), which have been introduced for a number of infectious diseases [7-14]. The rapid turnaround times of POCTs are potentially beneficial for making decisions in a variety of situations: isolation of infectious patients (and de-isolation of non-infectious ones); avoidance of unnecessary hospitalization; avoidance of unnecessary 
treatment (including reduced length of therapy); and improved selection of antimicrobial therapy (e.g., using a more appropriate, narrower spectrum agent) [7].

There are few reports in the literature of efforts to reduce laboratory turnaround times for $C$. difficile testing. Verdoorn and colleagues assessed the effect of telephoning out positive C. difficile results on the time to ordering antimicrobial therapy, which was reduced from a mean of 11.9-3.6 h [15]. Barbut and colleagues noted that changing their laboratory testing from a cytotoxicity assay to either PCR alone or in combination with glutamate dehydrogenase (GDH) led to a significant reduction in turnaround time from a mean of 3.5-0.55 days. This was associated with a reduction in unnecessary empirical therapy, length of stay and a non-significant reduction in mortality [16].

The present literature on real-world assessment of POCT for infectious diseases is limited [9] and no studies have evaluated $C$. difficile testing in a near-patient environment. This is mostly due to the lack of commercially available assays that can be used for this purpose. However, several manufacturers are developing highly sensitive molecular-based tests that could be implemented at POCT. These tests have been proposed or evaluated in a number of infectious diseases e.g., MRSA [10], influenza [17], sexually transmitted infections [11], group B Streptococcus [12], tuberculosis [13] and HIV [14].

The authors performed a feasibility study to evaluate acceptability, ease of use, change in turnaround time and clinical utility of a rapid, polymerase chain reaction (PCR) POCT (Cepheid GeneXpert $^{\circledR}$, Sunnyvale, California, USA) in three older persons' wards and two intensive care units (ICUs).

\section{METHODS}

\section{Setting}

The study was conducted in a central London academic hospital, with 1,100 beds, including 180 individual isolation rooms. Patients admitted with or who develop diarrhea and/or vomiting are placed in these rooms (with private bathroom), and kept there until at least $48 \mathrm{~h}$ following return to normal bowel habit. If this is not possible, the patient is placed in a cohorted, or an otherwise unoccupied, bay. Clinicians are advised to investigate all cases of diarrhea (two or more liquid stools in $24 \mathrm{~h}$ ). The rate of CDI in our institution between April 2011 and March 2012 was 32.2 cases per 100,000 occupied bed days (OBD). This compares to a national rate of 61.9 cases per 100,000 OBD for the same period.

The UK does not define technical criteria for assessing the suitability of POCT; however, there are local guidelines which are overseen by a Point of Care Committee in our hospital [18].

The study was conducted between March 2011 and January 2013 (22 months) in two settings; three adjacent older persons' wards comprising a total of 85 beds, and two adjacent ICUs comprising a total of 30 beds. Comparator wards, consisting of one older persons' ward and one ICU, had access only to laboratorybased testing and were used to compare study wards to investigate potential clinical utility.

Members of staff were asked to test any patient with clinically significant diarrhea for CDI using the POCT $\left(\right.$ GeneXpert $\left.{ }^{\circledR}\right)$; the residual sample was then tested in the centralized laboratory. The GeneXpert ${ }^{\circledR}$ system (Cepheid, Sunnyvale, California, USA) is an automated, disposable cartridge based, real-time PCR assay 
which detects the genes for toxin B $(t c d B)$, binary toxin (cdt) and a point mutation associated with PCR ribotype 027. A positive for the toxin $\mathrm{B}$ target indicates that toxigenic $C$. difficile has been detected; the two other targets provide information about the presence of presumptive ribotype 027. Two GeneXpert ${ }^{\circledR}$ systems were placed in the utility rooms of the three adjacent older persons' wards. The ICU has its own co-located satellite laboratory, capable of performing a range of near-patient tests, into which a GeneXpert ${ }^{\circledR}$ system was placed.

The residual stool sample was sent to the centralized laboratory for testing in parallel using a two-step algorithm [19] which comprised GDH (GDH Chek-60, TechLab, Blacksburg, Virginia, USA), with PCR $\left(\right.$ GeneXpert $^{\circledR}$ ) as a confirmatory step for positives. Results from both testing methods together with turnaround times (from point of sample requesting to availability of result) were compared using the same sample.

\section{Compliance with Ethics Guidelines}

All procedures followed were in accordance with the ethical standards of the responsible committee on human experimentation (London City and East Research Ethics Committee) and with the Helsinki Declaration of 1975, as revised in 2000 and 2008. Informed consent was obtained from all patients for being included in the study.

\section{Staff Training}

Nurses, healthcare assistants (older persons' wards) and laboratory technicians (ICUs) were trained to use the POCT system by a research nurse. This generally took around $1 \mathrm{~h}$ and was done in small groups. Training consisted of a demonstration followed by direct observation of each staff member to ensure competence. Competent staff members were provided with a password to operate the GeneXpert ${ }^{\circledR}$ system. Additional training was provided to those requiring it. The research nurse was available between the hours of 9 am and $5 \mathrm{pm}$, Monday to Friday, to assist with any problems and train new staff members.

\section{Connectivity and Results}

The GeneXpert ${ }^{\circledR}$ systems were networked using Synapse software (Systelab Technologies S.A., Barcelona, Spain). This allowed real-time monitoring of test results and errors on all GeneXpert $^{\circledR}$ systems. The analyzers were not interfaced directly with either the Laboratory Information Management System or the Electronic Patient Record. The GeneXpert ${ }^{\circledR}$ analyzers were connected to printers, which automatically printed out individual patient results upon test completion. Staff members on older persons' wards were instructed to insert this into the patient's clinical notes; staff in ICU manually transferred the result to the Electronic Patient Record. Additionally, whenever any sample tested positive, an immediate automated email alert was sent to the study team and service infection control nurses from 9 am to $5 \mathrm{pm}$, Monday to Friday. Outside of these hours, infection control advice was provided by an infectious diseases/ microbiology physician. This allowed immediate notification of a case and subsequent infection control interventions to be implemented before the centralized laboratory testing result became available. Clinical staff were instructed to act upon the results as they would have had the sample been processed in the centralized laboratory. 


\section{Clinical Utility}

Patients who underwent testing with the POCT were age and sex matched with patients tested for CDI on non-study wards (older persons' ward or ICU) where POCT testing was not available. These groups were compared to determine any differences in length of stay, 30-day all-cause mortality and requesting of certain ancillary investigations e.g., stool culture, norovirus testing, radiological investigations etc.

\section{Acceptability and Ease of Use}

A questionnaire was designed to gauge users' experience and opinions on the POCT. A fivepoint scale was used to assess level of agreement with five statements covering ease of use, acceptability, turnaround time, and effect on bed management.

\section{RESULTS}

The study period lasted for 22 months (March 2011 to January 2013). During this time, a total of 330 patients were tested by the POCT; 97 (29\%) POCTs were performed on the older persons' wards and 233 (71\%) on ICU. A total of 335 POCTs were performed; 100 tests on the 97 elderly patients and 235 tests were performed on the 233 ICU patients.

A total of 76 older persons' staff were trained, comprising of 17 healthcare assistants with no formal qualifications, 46 junior or student nurses and 13 senior nurses. Each older persons' staff member processed an average of 1.3 tests. A total of 15 ICU laboratory technicians were trained, each processing an average of 18 tests.
The majority of POCTs performed on older persons' wards were undertaken between the hours of midday and $9 \mathrm{pm}(82 \%)$. This figure was lower for those performed in ICU (61\%). Figure 1 shows times of sample testing on the older persons' wards and ICUs.

\section{Turnaround Time}

The median total turnaround time for laboratory-based testing (from the point of test ordering to the point of result availability) was $18 \mathrm{~h}$, with a median laboratory analytical turnaround time of $9.1 \mathrm{~h}$. The majority of the time difference was accounted for by sample transportation. The median total turnaround time for all samples tested by POCT was $1.85 \mathrm{~h}$. The median turnaround time for POC tests processed on ICU (2.35 h) was slightly longer than that for tests processed on older persons' wards $(0.83 \mathrm{~h})$.

\section{Agreement with Laboratory Testing}

Of the 335 samples that were tested using the POCT, 20 (6\%) were either not received by the laboratory or there was insufficient material to perform further testing. Of the remaining 315 samples, 274 (87\%) were negative by both POCT and laboratory-based GDH, and 15 (4.8\%) were negative by POCT, positive by laboratory-based GDH but negative by laboratory-based PCR; these samples were considered to be non-discrepant. The remaining $26(8.2 \%)$ samples were positive by POCT; of these 20 were also laboratory-based GDH and PCR positive (considered nondiscrepant) and 6 were laboratory-based GDH negative (considered discrepant). Overall agreement was $98.1 \%$. In total, there were 6 (1.9\%) discrepant samples with a mean cycle 


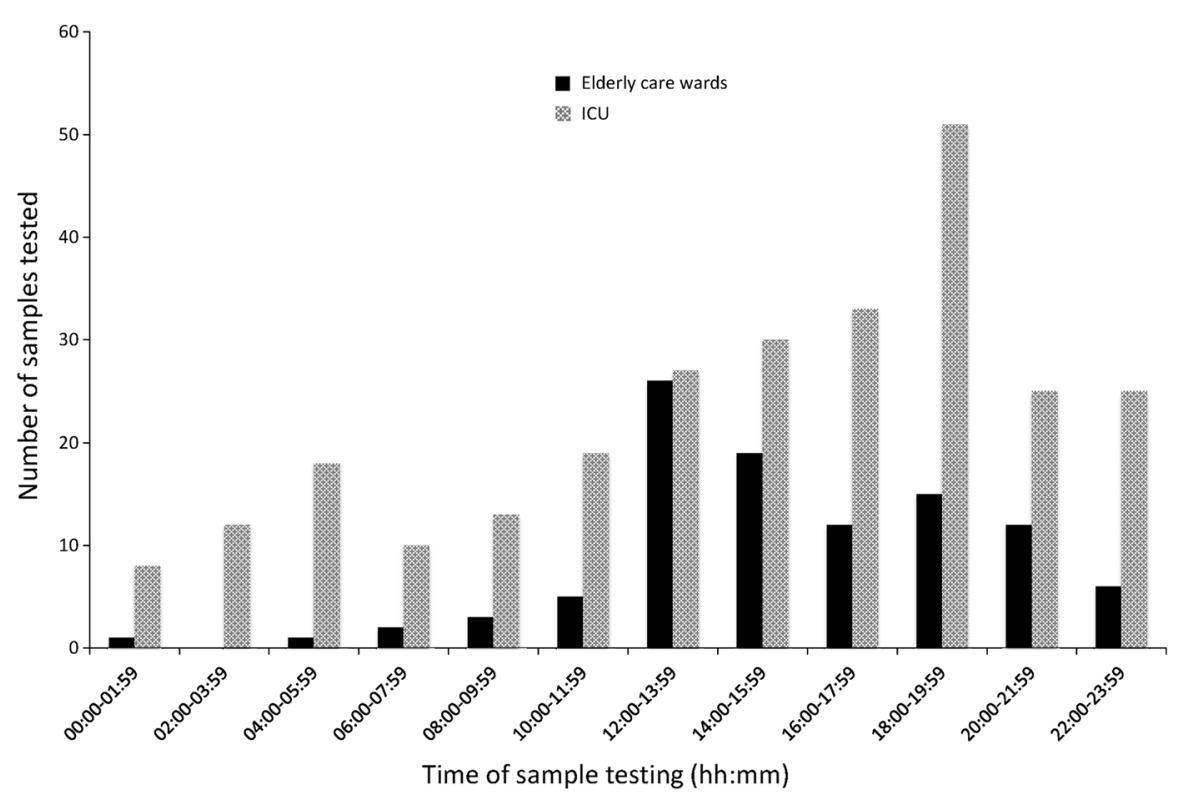

Fig. 1 Time of sample processing for POCT for older persons' wards (black) and ICU (gray). ICU Intensive care unit, POCT point-of-care test

threshold (Ct) value of 32.9. The maximum valid $\mathrm{Ct}$ for the toxin B target is 37 . Discrepant samples were more likely to occur on elderly wards $(n=3,3.9 \%$ of those tested) than ICU ( $n=3,1.3 \%$ of those tested), although this was not significant.

\section{Processing Errors}

Overall 20/335 (6\%) processing errors were encountered where a result was not obtained. These resulted from a variety of user and platform errors and were greatest in the first few months of the study (ten $(20.4 \%)$ errors in 49 tests performed in quarter one compared with two $(3.3 \%)$ errors in 61 tests performed in quarter five). During the second half of the study, an updated GeneXpert ${ }^{\circledR}$ cartridge was $^{-}$ introduced by the manufacturer, which had pre-filled reagents; this further simplified assay setup and reduced hands on time, although this did not have any effect on the number of processing errors. Overall, significantly more processing errors occurred on the older persons' wards $13 / 102(12.7 \%)$ than on ICU 7/271 (2.6\%) $p=<0.001$.

\section{Clinical Utility}

The mean age of all patients tested with the POCT was 66 years; with a lower mean age in the ICU patients (59 years) compared with older persons' patients (85 years). A greater proportion of patients tested positive in the older persons' wards $(14.4 \%$ and $17.4 \%$ of those tested by the POCT and the laboratory-based test, respectively) compared with ICU patients (6.9\% and $6.6 \%$ of those tested by the POCT and the laboratory-based test, respectively).

Overall, most patients were tested well into their hospital admission (mean of 16 days following admission). This corresponds with a high proportion of patients being classified as having hospital-associated diarrhea (onset of symptoms on day 3 or more following admission). However, of the older persons' 
patients, significantly more had hospital onset diarrhea in those tested with the laboratorybased test only (97\% compared with $84 \%$ for those were tested with the POCT).

Differences in patient demographics, ancillary clinical investigations and outcomes between patients tested by POCT and those in comparator wards tested in the laboratory are shown in Table 1. There were no significant differences in terms of length of stay and allcause mortality rates. Overall 30-day all-cause mortality rate was 5.1 per 1,000 inpatient days (25.3\%), which is slightly less than that reported elsewhere [20]. Older persons' patients who had POCT were significantly less likely to have a test requested for bacterial stool culture $(3.1 \%$ vs. $10.9 \% \quad p=0.044)$. This difference was not observed in the ICU patients. No other differences in ancillary test requesting were observed.

\section{Acceptability and Qualitative Feedback from Operators}

A user questionnaire was completed by 85 staff members in two phases (40 in phase one and 45 in phase two, following the introduction of the new GeneXpert ${ }^{\circledR}$ cartridges). Staff were permitted to participate in both phases. Sixtysix respondents $(78 \%)$ were older persons' staff and 19 (22\%) were ICU staff. All ICU staff in both rounds agreed that the test was easy to perform, compared with $76 \%$ of older persons' staff. The proportion of older persons' staff who agreed with this comment was no different in either phase of the questionnaire. All ICU respondents and $88 \%$ of older persons' respondents agreed that POCT results were available faster than laboratory testing. Seventy-six percent of ICU respondents liked being able to perform the test themselves and $94 \%$ felt it was an acceptable part of their role.
This compares with $86 \%$ and $80 \%$, respectively, in older persons' respondents. 95\% of ICU respondents and $86 \%$ of older persons' respondents thought that the test had helped them to manage beds more effectively (Fig. 2).

\section{DISCUSSION}

Diarrhea and CDI are major infection control challenges for hospitals and clinicians must decide on the most efficient use of scarce resources. Laboratory-based testing for $C$. difficile is sometimes slow but POCT could provide a faster result.

The data show that use of this POCT system is feasible in both the older persons' wards and the ICUs studied. However, more problems were encountered in the older persons' wards (more discrepant results and more processing errors). Although most older persons' staff reported that the test was easy to perform, this staff group are unfamiliar with carrying out this type of procedure. The ICU technicians were much more familiar with basic laboratory processes and this may account for the lower number of discrepant results and processing errors. The number of errors did not appear to decrease after the introduction of the updated GeneXpert ${ }^{\circledR}$ cartridge.

The six discrepant samples raise the possibility of contamination during assay preparation; all had relatively high Ct values. During training, users were educated about the importance of keeping the equipment and work areas clean and the disposable, single use nature of the cartridges and minimal sample handling to reduce the risk of contamination.

The processing errors could be related to the low volumes of tests being performed by any one individual staff member (particularly for the older persons' staff who processed an average of just one test each for the duration 


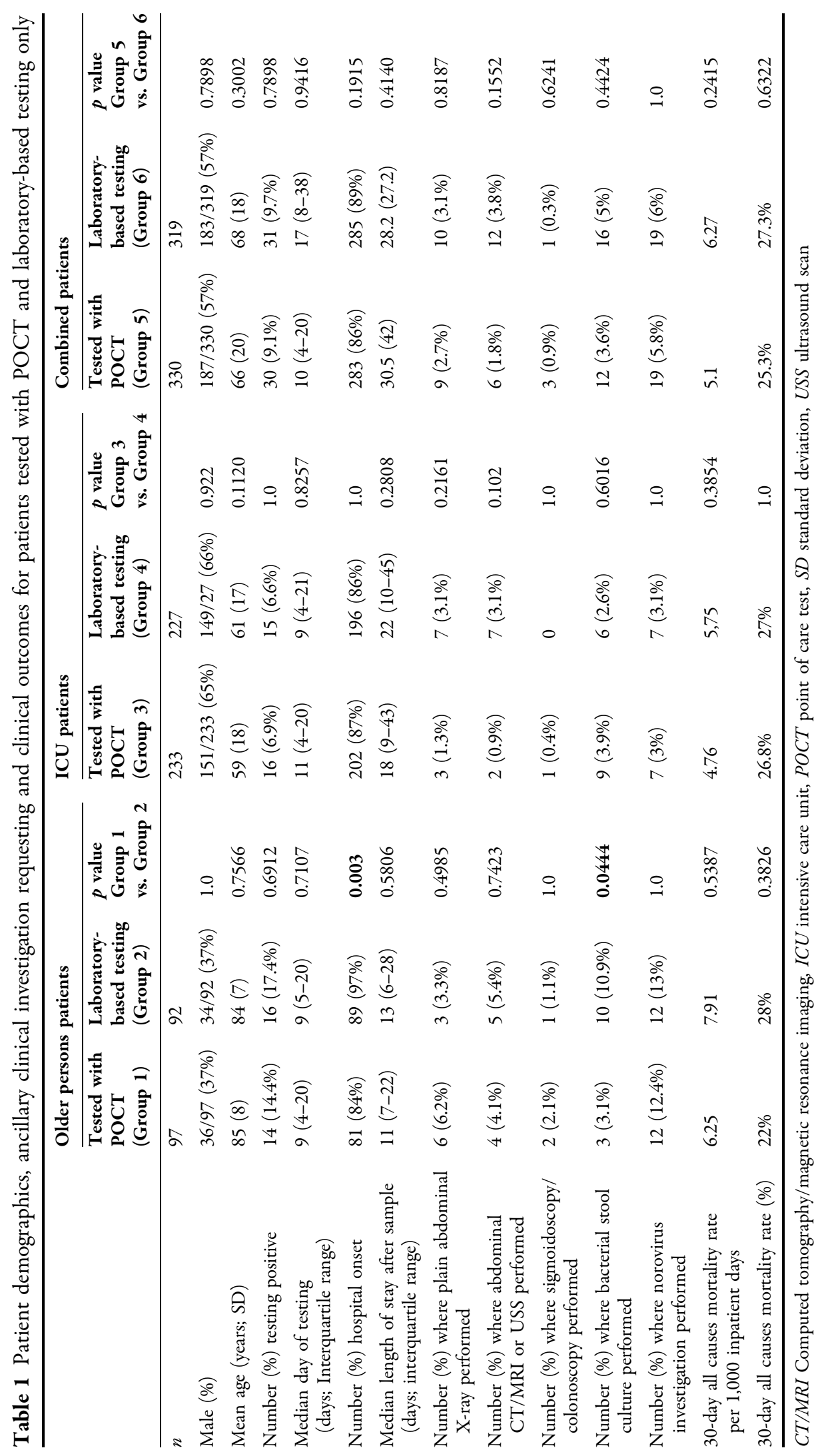




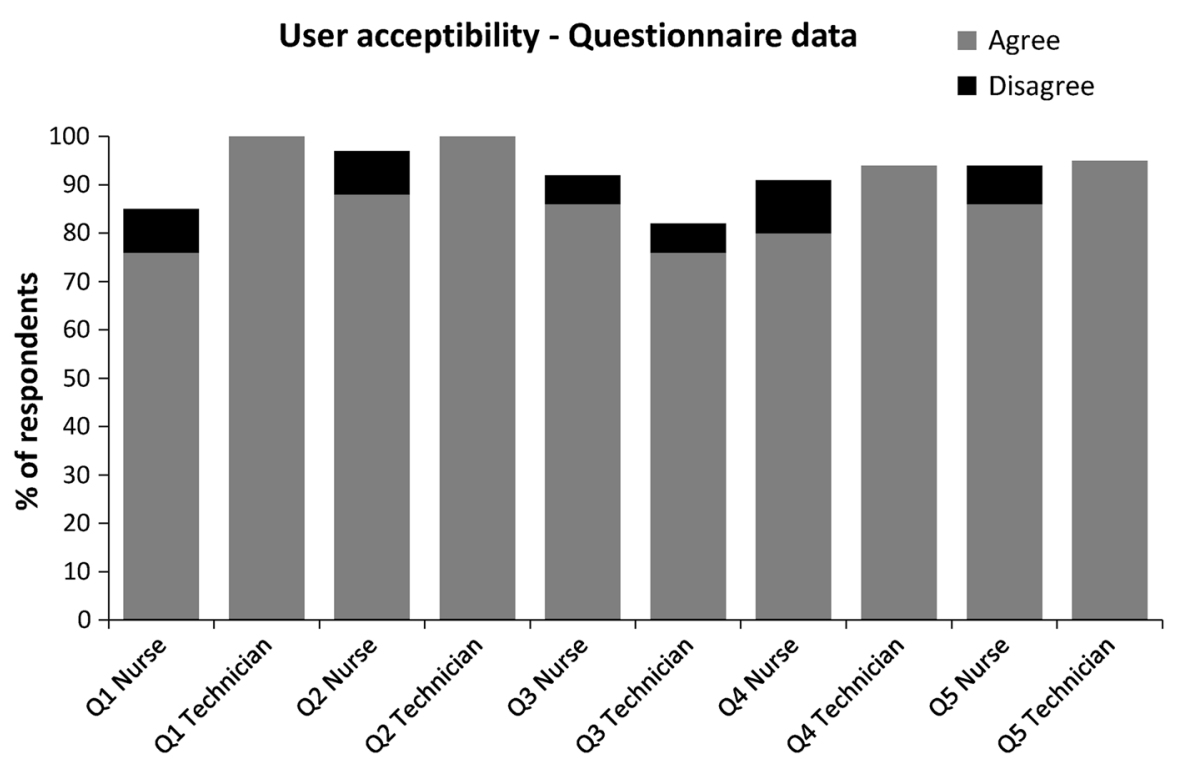

Fig. 2 Acceptability and ease of use. A total of 66 older persons' staff and 19 ICU laboratory technicians completed a user questionnaire, asking the level of agreement or disagreement with five statements based on a scale of 1 (completely agree) to 5 (completely disagree). The questions were as follows: (1) the POCT is easy to perform, (2) results from the POCT are available faster than the laboratory-based test, (3) I like being able to perform the POCT myself, (4) performing the POCT is an acceptable part of my role, (5) the POCT results have allowed better management of beds. ICU Intensive care unit, POCT point-of-care test of the study). A higher throughput of tests may have helped staff members to confidently recall how to perform the procedure. A more successful model of testing may be to make use of staff that are more familiar with laboratory procedures in a dedicated satellite POC laboratory [7]. Cohen-Bacrie and colleagues describe this model in their Marseilles hospitals and were able to achieve turnaround times between 0.5 and $3.5 \mathrm{~h}$ for a range of 23 POCTs of varying complexity [7].

Gray and colleagues found that assigning responsibility for Group B Streptococcus testing in laboring women to a relatively small group of staff ensured that each tester undertook enough testing to maintain competency [12]. With any POCT, there is a need for staff performing the test to be trained and competent in appropriate documentation, sample collection, performing the test and result interpretation. Failure to do this can have adverse outcomes in terms of assay performance [9].

Most tests were performed during the afternoon or early evening on the older persons' wards, whereas tests were performed throughout the day and night on the ICU. The numbers of nursing staff on the older persons' wards was lower through the night shift, but remained stable on the ICU. Patients may also be less willing to report diarrhea during the night and many patients on ICU were fitted with bowel managers making access to stool samples easier. In a study of POC testing for Group B Streptococcus in a UK delivery suite, Gray and colleagues found that testing increasingly became confined to normal working hours, when laboratory staff were available to assist [12].

The turnaround time of the POCT was significantly faster compared with laboratory- 
based testing (1.85 vs. $18 \mathrm{~h}$, respectively). Sample transportation caused a significant delay in our institution, batching of samples testing in the centralized laboratory also added on additional time, even when samples were tested twice per day. Although the turnaround time was significantly reduced, there were no discernable effects of the POCT on clinical utility other than a reduction in ancillary bacterial culture testing. This is likely to be a minimal cost saving and does not offset the significant costs of running the POCT. The numbers in this study were modest and the study may be insufficiently powered to detect any changes in clinical outcomes between those tested with POCT compared with those tested by laboratory-based testing. Future studies should look at other outcomes such as severity of disease, time to anti-C. difficile therapy (or deescalation of empiric therapy) and use of isolation facilities. It would be prudent to bear in mind, however, that a negative result for $C$. difficile does not necessarily mean that the patient can be removed from single room isolation, since the symptoms could be due to another infectious cause such as norovirus. Ideally the patient would be tested for a range of infectious agents to be confident that they do not pose a risk of cross transmission before deisolating [1].

UK and European guidance recommends testing for CDI using a two-step algorithm with either GDH or a molecular test as a first stage and confirming any positives with a toxin enzyme immunoassays (EIA) [21, 22]. This study was conceived and carried out before this guidance was published and there is still debate about the clinical interpretation of PCR positive tests in diarrheal patients [23].

Given the current testing guidelines endorsed by Public Health, England and European Society of Clinical Microbiology and
Infectious Diseases (ESCMID), perhaps there could be additional value of this assay in screening newly admitted patients for colonization. Asymptomatic carriage is widespread amongst hospital inpatients [24] and potential transmission from this group has already been demonstrated [25]. Peri-rectal swabs could provide a more convenient and acceptable sample type for screening patients [26]. The practice of screening for carriage is not widely practiced, however, modeling has shown that this approach may be cost effective [27].

Financial costs were not evaluated in this study. However, when deciding to implement a POCT, it is important to consider the often hidden costs of support from a local accredited laboratory, and costs of training and maintenance; these should be measured in any future evaluation.

\section{CONCLUSION}

This study demonstrates that POCT using the GeneXpert ${ }^{\circledR}$ system is feasible and acceptable to nursing staff and technicians working within the two extremes of these hospital-based settings. The assay has already been used in a variety of settings including in resource poor countries [28, 29]. These types of tests are becoming increasingly more common and it is important that they are assessed in the environment for which they are intended with high-quality clinical utility studies, which also evaluate cost effectiveness.

\section{ACKNOWLEDGMENTS}

We are grateful to the staff of the ICUs and older persons' wards who contributed to the study. This work was funded with a Grant from The Technology Strategy Board (Swindon UK) and 
by the National Institute for Health Research (NIHR) comprehensive Biomedical Research Centre award to Guy's and St Thomas' NHS Foundation Trust in partnership with King's College London. Article processing charges were funded by Cepheid Europe (Maurens-Scopont, France). All named authors meet the ICMJE criteria for authorship for this manuscript, had full access to all of the data in this study, take complete responsibility for the integrity of the data and accuracy of the data analysis, and have given final approval for the version to be published.

Conflict of interest. A Postulka is an employee of Cepheid Europe. SD Goldenberg, KN Bisnauthsing, A Patel, D Wyncoll, GL French and $\mathrm{R}$ Schiff declare no conflict of interest.

Compliance with ethics guidelines. All procedures followed were in accordance with the ethical standards of the responsible committee on human experimentation (London City and East Research Ethics Committee) and with the Helsinki Declaration of 1975, as revised in 2000 and 2008. Informed consent was obtained from all patients for being included in the study.

Open Access. This article is distributed under the terms of the Creative Commons Attribution Noncommercial License which permits any noncommercial use, distribution, and reproduction in any medium, provided the original author(s) and the source are credited.

\section{REFERENCES}

1. Halligan E, Edgeworth J, Bisnauthsing K, Bible J, Cliff P, Aarons E, Klein J, Patel A, Goldenberg S.
Multiplex molecular testing for management of infectious gastroenteritis in a hospital setting: a comparative diagnostic and clinical utility study. Clin Microbiol Infect. 2014;20(8):460-7.

2. McDonald LC, Killgore GE, Thompson A, Owens RC Jr, Kazakova SV, Sambol SP, Johnson S, Gerding DN. An epidemic, toxin gene-variant strain of Clostridium difficile. N Engl J Med. 2005;353: 2433-41.

3. Kundrapu S, Jury LA, Sitzlar B, Sunkesula VC, Sethi AK, Donskey CJ. Easily modified factors contribute to delays in diagnosis of Clostridium difficile infection: a cohort study and intervention. J Clin Microbiol. 2013;51:2365-70.

4. Culbreath K, Ager E, Nemeyer RJ, Kerr A, Gilligan $\mathrm{PH}$. Evolution of testing algorithms at a university hospital for detection of Clostridium difficile infections. J Clin Microbiol. 2012;50:3073-6.

5. Sunkesula VC, Kundrapu S, Jury LA, Deshpande A, Sethi AK, Donskey CJ. Potential for transmission of spores by patients awaiting laboratory testing to confirm suspected Clostridium difficile infection. Infect Control Hosp Epidemiol. 2013;34:306-8.

6. Saade E, Deshpande A, Kundrapu S, Sunkesula VC, Guerrero DM, Jury LA, Donskey CJ. Appropriateness of empiric therapy in patients with suspected Clostridium difficile infection. Curr Med Res Opin. 2013;29:985-8.

7. Cohen-Bacrie $S$, Ninove L, Nougairède A, Charrel R, Richet H, Minodier P, Badiaga S, Noël S, La Scolla B, de Lamballerie $X$, Drancourt $M$, Raoult D. Revolutionizing clinical microbiology laboratory organization in hospitals with in situ point-ofcare. PLoS One. 2011;6:e22403.

8. Fournier PE, Drancourt M, Colson P, Rolain JM, La Scola B, Raoult D. Modern clinical microbiology: new challenges and solutions. Nat Rev Microbiol. 2013;11:574-85.

9. Moore C. Point-of-care tests for infection control: should rapid testing be in the laboratory or at the front line? J Hosp Infect. 2013;85:1-7.

10. Brenwald NP, Baker N, Oppenheim B. Feasibility study of a real-time PCR test for meticillin-resistant Staphylococcus aureus in a point of care setting. J Hosp Infect. 2010;74:245-9.

11. Turner KM, Round J, Horner P, McLeod J, Goldenberg S, Deol A, Adams EJ. An early evaluation of clinical and economic costs and benefits of implementing point of care NAAT tests for Chlamydia trachomatis and Neisseria gonorrhoea in genitourinary medicine clinics in England. Sex Transm Infect. 2014;90:104-11. 
12. Gray JW, Milner PJ, Edwards EH, Daniels JP, Khan KS. Feasibility of using microbiology diagnostic tests of moderate or high complexity at the point-of-care in a delivery suite. J Obstet Gynaecol. 2012;32:458-60.

13. Theron G, Zijenah L, Chanda D, Clowes P, Rachow A, Lesosky M, Bara W, Mungofa S, Pai M, Hoelscher $\mathrm{M}$, et al. Feasibility, accuracy, and clinical effect of point-of-care xpert MTB/RIF testing for tuberculosis in primary-care settings in Africa: a multicentre, randomised, controlled trial. Lancet. 2014;383: 62073-5.

14. Burns F, Edwards SG, Woods J, Haidari G, Calderon Y, Leider J, Morris S, Tobin R, Cartledge J, Brown M. Acceptability, feasibility and costs of universal offer of rapid point of care testing for HIV in an acute admissions unit: results of the RAPID project. HIV Med. 2013;14:10-4.

15. Verdoorn BP, Orenstein R, Wilson JW, Estes LL, Wendt RF, Schleck CD, Harmsen WS, Nyre LM, Patel R. Effect of telephoned notification of positive Clostridium difficile test results on the time to the ordering of antimicrobial therapy. Infect Control Hosp Epidemiol. 2008;29:658-60.

16. Barbut F, Surgers L, Eckert C, Visseaux B, Cuingnet M, Mesquita C, Pradier N, Thiriez A, Ait-Ammar N, Aifaoui A, et al. Does a rapid diagnosis of Clostridium difficile infection impact on quality of patient management? Clin Microbiol Infect. 2014;20:136-44.

17. Babin SM, Hsieh YH, Rothman RE, Gaydos CA. A meta-analysis of point-of-care laboratory tests in the diagnosis of novel 2009 swine-lineage pandemic influenza A (H1N1). Diagn Microbiol Infect Dis. 2011;69:410-8.

18. Medical Devices Agency. Management and use of IVD point-of-care test devices. London: Medical Devices Agency 2003; MDA DB2002(03).

19. Goldenberg SD, Cliff PR, Smith S, Milner M, French GL. Two-step glutamate dehydrogenase antigen real-time polymerase chain reaction assay for detection of toxigenic clostridium difficile. J Hosp Infect. 2010;74:48-54.

20. Planche TD, Davies KA, Coen PG, Finney JM, Monahan IM, Morris KA, O'Connor L, Oakley SJ, Pope CF, Wren MW, et al. Differences in outcome according to Clostridium difficile testing method: a prospective multicentre diagnostic validation study of C. difficile infection. Lancet Infect Dis. 2013;13: 936-45.
21. Crobach MJ, Dekkers OM, Wilcox MH, Kuijper EJ. European Society of Clinical Microbiology and Infectious Diseases (ESCMID): data review and recommendations for diagnosing Clostridium difficile-infection (CDI). Clin Microbiol Infect. 2009; 15:1053-66.

22. Updated guidance on the diagnosis and reporting of Clostridium difficile. Best Practice Guideline 17215. Department of Health 2012, Mar 6. https:// www.gov.uk/government/publications/updatedguidance-on-the-diagnosis-and-reporting-of-clostridium-difficile.

23. Wilcox MH, Planche T, Fang FC, Gilligan P. What is the current role of algorithmic approaches for diagnosis of Clostridium difficile infection? J Clin Microbiol. 2010;48:4347-53.

24. Guerrero DM, Becker JC, Eckstein EC, Kundrapu S, Deshpande A, Sethi AK, Donskey CJ. Asymptomatic carriage of toxigenic Clostridium difficile by hospitalized patients. J Hosp Infect. 2013;85:155-8.

25. Curry SR, Muto CA, Schlackman JL, Pasculle AW, Shutt KA, Marsh JW, Harrison LH. Use of multilocus variable number of tandem repeats analysis genotyping to determine the role of asymptomatic carriers in Clostridium difficile transmission. Clin Infect Dis. 2013;57:1094-102.

26. Curry SR, Schlackman JL, Hamilton TM, Henderson TK, Brown NT, Marsh JW, Shutt KA, Brooks MM, Pasculle AW, Muto CA, et al. Peri-rectal swab surveillance for Clostridium difficile using selective broth pre-amplification and real-time PCR detection of $t c d B$. J Clin Microbiol. 2011;49:378893.

27. Bartsch SM, Curry SR, Harrison LH, Lee BY. The potential economic value of screening hospital admissions for Clostridium difficile. Eur J Clin Microbiol Infect Dis. 2012;31:3163-71.

28. Bouricha M, Samad MA, Levy PY, Raoult D, Drancourt M. Point-of-care syndrome-based, rapid diagnosis of infections on commercial ships. J Travel Med. 2014;21:12-6.

29. Pai NP, Vadnais C, Denkinger C, Engel N, Pai M. Point-of-care testing for infectious diseases: diversity, complexity, and barriers in low- and middle-income countries. PLoS Med. 2012;9: e1001306. 